\title{
Adaptation to Multifocal and Monovision Contact Lens Correction
}

\author{
Paulo R.B. Fernandes*, Helena I. F. Neves ${ }^{\dagger}$, Daniela P. Lopes-Ferreira ${ }^{\star}$, Jorge M.M. Jorge*, \\ and José M. González-Meijome*
}

\begin{abstract}
Purpose. To compare visual performance with the Biofinity multifocal (MF) contact lens with monovision (MV) with the Biofinity single-vision contact lens.

Methods. A crossover study of 20 presbyopic patients was conducted. Patients were randomized first into either an MF or an MV lens for 15 days for each modality, with a washout period between each lens type. Measurements included monocular and binocular high- and low-contrast logarithm of the minimum angle of resolution visual acuity (VA) at distance and near visions, binocular distance contrast sensitivity function, and near stereoacuity.

Results. At 15 days, patients lost fewer than two letters (half a line of VA) of binocular distance and near VA, with the MF and MV lens under high- and low-contrast conditions $(P>0.05$ for both comparisons). No statistically significant differences were seen in binocular VA at near or distance with either lens. However, the monocular distance VA improved significantly in the nondominant eye, with the MF lens by one line over the 15-day period under high-contrast $(P=0.023)$ and lowcontrast $(P=0.035)$ conditions; this effect was not seen with the MV lens. Contrast sensitivity function was within the normal limits with both lenses. The stereoacuity was significantly $(P<0.01)$ better with MF than with MV.

Conclusions. Multifocal contact lens correction provided satisfactory levels of VA comparable with MV without compromising stereoacuity in this crossover study. The near vision significantly improved in the dominant eye, and the distance vision improved in the nondominant eye from 1 to 15 days with the MF lens, suggesting that patients adapted to the multifocality overtime, whereas this was not true for MV.
\end{abstract}

(Optom Vis Sci 2013;90:228-235)

Key Words: monovision, multifocal contact lenses, logMAR visual acuity, stereoacuity, subjective comfort and ability

$\mathrm{F}$ or the last 2 decades, there has been a progressive aging of the population, with a corresponding increase in the proportion of the world population that becomes presbyopic. Providing satisfactory procedures to correct presbyopia with contact lenses that offer instant and optically clear distance and near vision is the biggest challenge for the industry and contact lens practitioners. The number of contact lens wearers requiring presbyopic correction has also grown significantly in recent years. Although there is considerable variation among countries ${ }^{1,2}$ (ranging from $0 \%$ to $79 \%$ of all soft lens fittings in patients $>45$ years), only approximately $29 \%$ and $8 \%$ of presbyopes are corrected with multifocal

${ }^{*} \mathrm{OD}, \mathrm{PhD}$

${ }^{\dagger} \mathrm{OD}$

${ }^{\ddagger} \mathrm{OD}, \mathrm{MSc}$

Clinical and Experimental Optometry Research Laboratory (CEORLab), Center of Physics (Optometry), School of Sciences, University of Minho, Braga, Portugal (all authors).
(MF) or monovision (MV) modalities, respectively, whereas more than $60 \%$ of presbyopes are still being fitted with nonpresbyopic corrections. $^{2}$ Current techniques to correct presbyopia with contact lenses are based on one of three principles: MV, alternating vision, or simultaneous vision. Monovision ${ }^{3}$ involves fitting one eye for distance (typically the dominant eye) and one eye for near vision, whereas simultaneous vision designs provide concurrent clear vision at two or more distances by broadening the lens-eye system depth of focus. ${ }^{4}$ Although previous reports have reported success rates with MV between $59 \%$ and $67 \%,{ }^{3}$ significant decreases in stereopsis ${ }^{5,6}$ and vision-related quality of life have been reported. ${ }^{7}$ Recent studies have confirmed that the MF option offers similar or superior patient satisfaction by providing better stereoacuity and near range of clear vision ${ }^{5,8-10}$ as well as patient preference over MV. 6,11

The objective of this study was two-fold, that is, to establish if there is a difference in comfort and subjective satisfaction between the new Biofinity balanced MF silicone hydrogel (Comfilcon A) 
contact lens (CooperVision, Fairport, NY) and MV with the Biofinity single-vision contact lenses (CooperVision) and to compare the visual performances of MF and MV modalities using a series of visual assessments, including traditional visual acuity (VA)-based measures and subjective responses and task-based evaluations to assess as wide a range of visual experiences as possible to determine which modality better satisfies the needs of presbyopic patients.

\section{METHODS}

Twenty-three patients ( 8 men, 15 women; mean age, 48.7 (3.3) years; range, 45-57 years) were recruited at the Clinical and Experimental Optometry Research Laboratory of the University of Minho from the staff of the university who responded to an e-mail. The study followed the tenets of the Declaration of Helsinki; all participants provided informed consent after they received an explanation of the nature, procedures, and consequences of the study. A local review board approved the study. The inclusion criteria were a minimal age of 45 years, maximal spectacle astigmatism of $0.75 \mathrm{D}$ in either eye, best-corrected distance VA of at least $0.18 \log$ MAR in each eye, no binocular vision anomaly, no ocular or systemic disease, and no need for medication that might interfere with or contraindicate contact lens wear. Previous contact lens wear was not required, and 2 of the 23 subjects recruited had previous contact lens experience. However, no participant had previously worn presbyopic contact lenses.

Once the suitability of the subjects was confirmed, each was assigned randomly to be fitted with one of the two contact lenses. All subjects received the MF contact lenses, according to the manufacturer's fitting guidelines for the initial lens selection. No subject received a combination of add powers, and the near add power for the MV correction was matched with the near power of the MF modality.

The Biofinity MF contact lens is made of a silicone hydrogel material (Comfilcon A) that has a water content of $48 \%$, a total diameter of $14.0 \mathrm{~mm}$, and a base curve of $8.60 \mathrm{~mm}$. The balanced presbyopic design combines MF optics with one lens for distance viewing and the other lens for near viewing. This design combines spherical and aspheric optics and unique zone sizes to yield a "distance" lens for the dominant eye (center-distance design), which emphasizes distance vision, and a "near" lens for the nondominant eye (center-near design), which optimizes near vision. The distance lens has a spherical central zone $2.3 \mathrm{~mm}$ in diameter for distance vision, surrounded by a $5.0-\mathrm{mm}$ annular aspheric zone and an $8.5-\mathrm{mm}$ spherical annular zone both increasing in add power. In contrast, the near lens has a $1.7-\mathrm{mm}$ spherical central zone dedicated to near vision followed by a 5.0-mm aspheric annular zone and an $8.5-\mathrm{mm}$ spherical annular zone both with decreasing add power. Each distance and near lens design is available from +4.00 to -6.00 in 0.25 -D steps and in four add powers $(+1.00,+1.50,+2.00$, and +2.50 in stock or higher upon request). For MV, the Biofinity sphere was used, which is a single-vision lens (aspheric design) of the same material as the Biofinity MF lens.

The dominant eye was fitted with the distance lens, and the nondominant eye with the near lens. Dominance was identified using the "sensory dominance method" in which the patient looked to a line immediately below his or her best VA (highcontrast $\log$ MAR VA chart at $4 \mathrm{~m}$ ), and a +1.50 -D lens was placed alternately in front of each eye for a few seconds; the subject described which eye had more blurred vision under binocular conditions. With both contact lenses, the optimal distance and near VA and on-eye lens fit were ensured for each eye using standard optometric techniques. Patients were instructed on lens insertion, removal, and cleaning techniques. All patients received a lens care system of one-step hydrogen peroxide.

Once the fitting procedure was completed, subjects were asked to return 14 days later for a follow-up visit to evaluate the fit, vision, and comfort, and after a $48-\mathrm{h}$ washout period, for a refitting for the second pair. During each visit, we performed the following clinical assessments.

The distance VA was recorded at $4 \mathrm{~m}$ with a Logarithmic Visual Acuity Chart Early Treatment Diabetic Retinopathy Study (ETDRS) (Precision Vision, LaSalle, IL) under high-contrast (100\%; chart acuity test [CAT] No. 2110) and low-contrast (10\%; CAT No. 2153) conditions with a Cabinet Illuminator No. 2425 (Precision Vision). The near VA was recorded at a distance of $40 \mathrm{~cm}$ using the Logarithmic Visual Acuity Chart 2000 "New ETDRS" (Chart "1," CAT No. 2106), as recommended, for high-contrast (100\%) and with Chart "2" (CAT No. 2117) for low-contrast $(10 \%)$ conditions. All VA measurements were conducted under photopic conditions $\left(85 \mathrm{~cd} / \mathrm{m}^{2}\right)$. All VA values reported refer to high- or low-contrast distance VA (HCDVA or LCDVA, respectively), whereas HCNVA or LCNVA will be used for high- or lowcontrast near VA, respectively.

The near contrast sensitivity function (CSF) was recorded at $40 \mathrm{~cm}$, with and without level II Glare, using the Functional Acuity Contrast Test (FACT) incorporated in a Functional Visual Analyzer (Stereo Optical Co., Inc., Chicago, IL) for spatial frequencies of $1.5,3,6,12$, and 18 cycles per degree. This device allows fine control of the distance of the examination and luminance conditions and provides comparable values to the vision contrast test system VCTS 6500 (Vistech Consultants, Dayton, $\mathrm{OH}) .{ }^{12}$ The results correspond to the binocular distance CSF in logarithmic units.

The near stereoacuity was recorded at $40 \mathrm{~cm}$ using the Stereo Fly SO-001 (Stereo Optical Co.).

For subjective evaluation of visual comfort and ability, we used a visual satisfaction questionnaire consisting of multiple vertical visual analog scales that evaluated subjective comfort and handling, subjective visual performance, and subjective task performance. Subjective comfort and handling were graded from very uncomfortable to very comfortable and from very difficult to very easy, respectively; for subjective visual and task performance, patients were requested to rate their satisfaction regarding distance, intermediate, near, and high or dim/poor light visions and the degree of difficulty experienced with driving, recognizing faces, and near visual tasks such as reading small print, reading mail, using a computer, and doing fine handwork, among others. Scores ranged from 0 to 10 vertically-oriented visual analog scale, with a lower score indicating greater difficulty performing visual tasks. Subjects completed a questionnaire at the final visit for both contact lens modalities.

All clinical measurements of visual function were conducted monocularly and binocularly under consistent room illumination, 
and after the 48-h washout period, subjects then were refitted with the alternative lens type, and all procedures then were repeated for the second lens.

Statistical power analysis performed before the start of the study showed that an estimated final sample size of 20 subjects required a power ranging from 0.88 (to detect a 1.5 -point difference in subjective ratings with an SD of 2.0) to 0.92 (to detect differences of $0.08 \log$ MAR unit, i.e., -4 letters in HCDVA with an SD of $0.1,-1$ whole line), for a level of statistical significance of 0.05 . Statistical analysis was performed using SPSS for Windows software (version 19, SPSS, Inc., Chicago, IL). The normality of the data was checked using the Kolmogorov-Smirnov test. The nonparametric Friedman test was used to evaluate the statistical significance of VA differences over time, and when significant differences were indicated, a paired Wilcoxon signed rank-sum test was performed. The Wilcoxon signed-rank test was used to compare visual function and stereoacuity between the two contact lens types and to compare the subjective evaluations of visual comfort and ability. For CSF comparisons, the repeated-measures analysis of variance with post hoc correction (Bonferroni) for multiple comparisons was used. Differences were considered statistically significant when $P<0.05$.

\section{RESULTS}

Of the 23 patients enrolled, 3 were lost to follow-up, with no data beyond the 7-day visit with the first randomly assigned modality, and were excluded from the study. Of the 20 subjects who completed the study, 13 (65\%) were women and 8 (40\%) were myopic. All except two patients had never worn contact lenses. Of them, 35\% had progressive-add spectacle lenses, 30\% had near glasses, $10 \%$ were wearing single-vision soft contact lens, and $25 \%$ were single-vision spectacle wearers and did not use any near vision correction despite their presbyopic complaints. The mean (SD) distance manifest refraction (spherical equivalent) was $-0.91(2.25) \mathrm{D}$ (range, -6.88 to 1.63 ) in the dominant eye and $-0.83(2.35) \mathrm{D}$ (range, -7.38 to 2.00 ) in the nondominant eye. The mean add power was $1.53(0.58) \mathrm{D}$.

The results comparing visual function with the two lens types at the 15-day visit are shown in Table 1. The binocular distance VA was slightly better for MF compared with MV in all conditions studied; however, there were no significant differences between the contact lens modalities ( $P>0.05$ in all comparisons). Nevertheless, the monocular distance VA was significantly better with MF correction in the nondominant eye for HCDVA (0.16 vs. $0.26 \log$ MAR, $P<0.01)$ and LCDVA $(0.35$ vs. 0.43 $\log$ MAR, $P<0.01)$. Regarding the near VA, there were no significant differences in the binocular VA between MF and MV correction; however, the near VA was significantly better with the MF lens in the dominant eye in high-contrast $(0.20$ vs. 0.38 $\log$ MAR, $P<0.01)$ and low-contrast $(0.37$ vs. $0.53 \log$ MAR, $P<0.01)$ conditions.

During the 15 days of use, there were no significant changes in binocular HCDVA and LCDVA with the MF contact lenses $(P>0.05$, for high and low contrasts) or in MV correction $(P>0.05$, for high and low contrasts). There was a significant improvement in the distance VA for nondominant eyes with the MF lens in which the distance VA improved significantly by one line after 15 days under high-contrast $(P=0.023)$ and lowcontrast $(P=0.035)$ conditions. For near VA, there was also a

TABLE 1.

Summary of visual performance between the Biofinity multifocal contact lens and monovision correction at 15 days

\begin{tabular}{|c|c|c|c|c|c|}
\hline & & \multicolumn{3}{|c|}{ Mean (SD) } & \multirow[b]{2}{*}{$P$} \\
\hline & & Baseline & MV & MF & \\
\hline \multirow[t]{3}{*}{ HCDVA } & Binocular & $-0.10(0.10)$ & $-0.08(0.09)$ & $-0.09(0.08)$ & Overall, $0.342^{*}$ \\
\hline & Dominant eye & $-0.06(0.08)$ & $-0.06(0.08)$ & $-0.05(0.09)$ & Overall, $0.495^{*}$ \\
\hline & Nondominant eye & $-0.02(0.14)$ & $0.26(0.16)$ & $0.16(0.14)$ & $\begin{array}{l}\text { Overall, }<0.001^{\dagger} \\
M V / M F_{,}<0.001^{\dagger}\end{array}$ \\
\hline \multirow[t]{4}{*}{$\overline{\mathrm{LCDVA}}$} & Binocular & $0.06(0.08)$ & $0.11(0.08)$ & $0.11(0.06)$ & Overall, $0.270^{*}$ \\
\hline & Dominant eye & $0.11(0.09)$ & $0.14(0.07)$ & $0.14(0.06)$ & Overall, $0.339^{*}$ \\
\hline & Nondominant eye & $0.17(0.15)$ & $0.43(0.19)$ & $0.35(0.13)$ & Overall, $<0.001$ \\
\hline & & & & & $\mathrm{MV} / \mathrm{MF},<0.001^{\dagger}$ \\
\hline \multirow[t]{3}{*}{ HCNVA } & Binocular & $0.01(0.06)$ & $0.05(0.10)$ & $0.04(0.07)$ & Overall, $0.188^{*}$ \\
\hline & Dominant eye & $0.10(0.11)$ & $0.38(0.17)$ & $0.20(0.09)$ & $\begin{array}{l}\text { Overall, }<0.001 \\
\text { MV/MF }<0.001^{\dagger}\end{array}$ \\
\hline & Nondominant eye & $0.08(0.11)$ & $0.07(0.13)$ & $0.06(0.07)$ & Overall, 0.835 \\
\hline \multirow[t]{3}{*}{ LCNVA } & Binocular & $0.19(0.07)$ & $0.23(0.12)$ & $0.21(0.09)$ & Overall, $0.068^{*}$ \\
\hline & Dominant eye & $0.28(0.12)$ & $0.53(0.18)$ & $0.37(0.12)$ & $\begin{array}{l}\text { Overall, }<0.001^{*} \\
M V / M F,<0.001^{\dagger}\end{array}$ \\
\hline & Nondominant eye & $0.25(0.08)$ & $0.28(0.15)$ & $0.23(0.09)$ & Overall, $0.232^{*}$ \\
\hline
\end{tabular}

Significant differences are highlighted in italics.

*Friedman test.

${ }^{\dagger}$ Wilcoxon signed-rank test.

HCDVA, high-contrast distance visual acuity; HCNVA, high-contrast near visual acuity; LCDVA, low-contrast distance visual acuity; LCNVA, low-contrast near visual acuity; MV/MF = monovision/multifocal. 
A
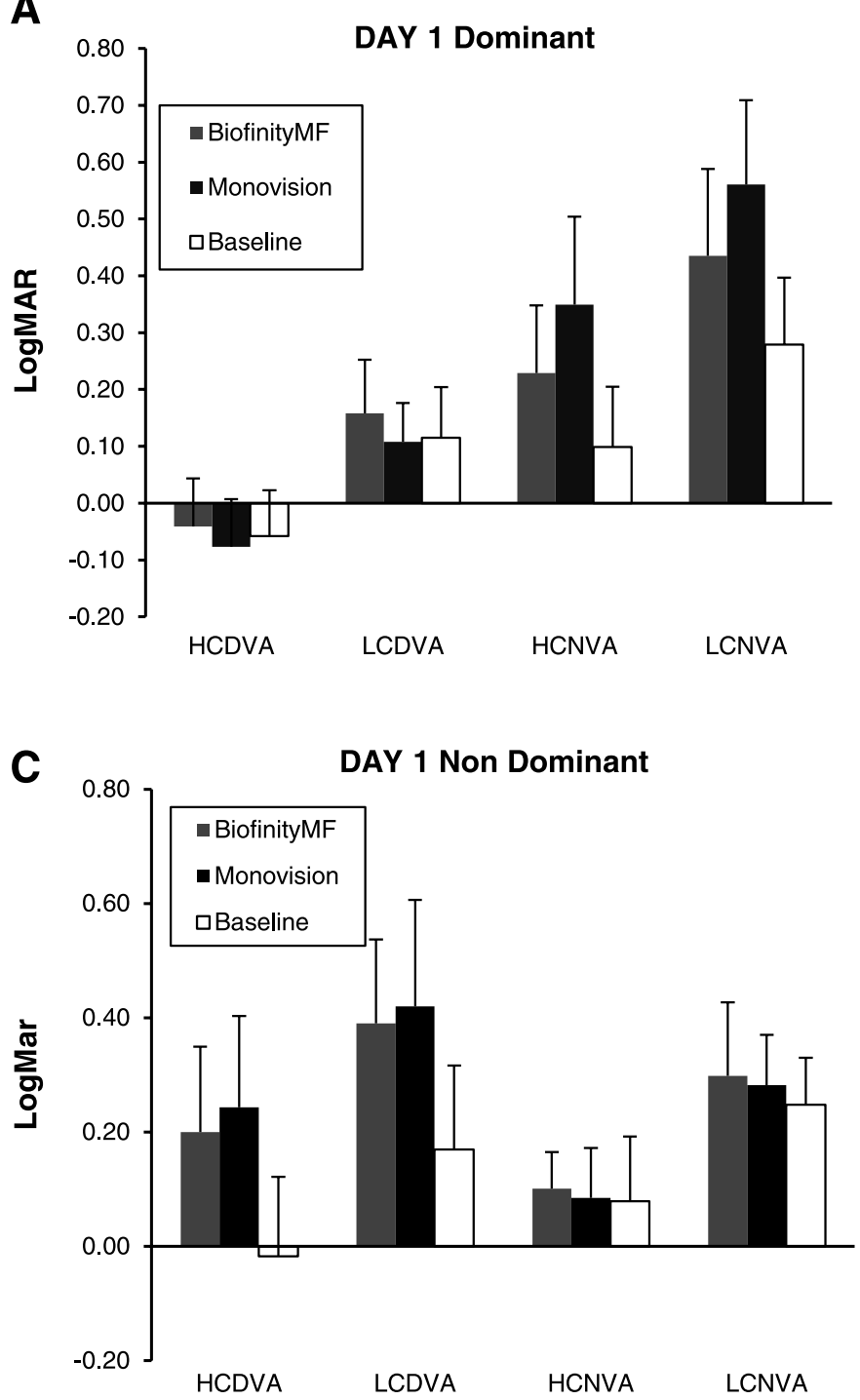

B
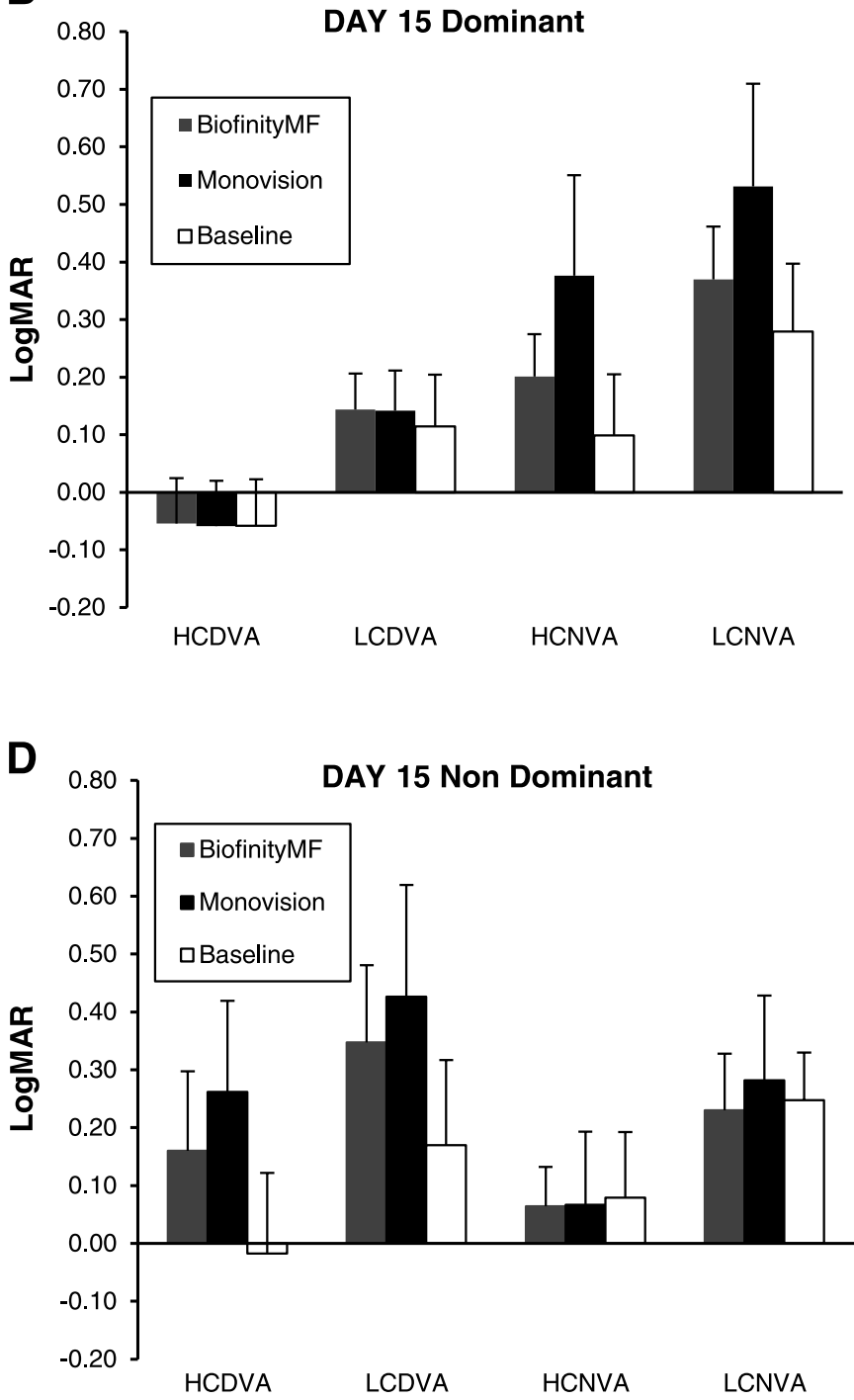

\section{FIGURE 1.}

Changes in VA (logMAR) from days 1 to 15 in dominant $(A, B)$ and nondominant $(C, D)$ eyes with each contact lens modality. The HCDVA and LCDVA contrast distance VA are significantly improved in the nondominant eyes with the MF modality. The same effect is seen for the HCNVA in the nondominant eye and in the low contrast near VA for the dominant and nondominant eyes.

significant improvement in HCNVA in the nondominant eye $(P=0.048)$, and in LCNVA, in the dominant $(P=0.026)$ and nondominant $(P=0.017)$ eyes (Fig. 1$)$.

There were no significant changes in the distance CSF with either correction compared with baseline or between both contact lens modalities ( $P>0.05$ for all spatial frequencies) (Fig. 2). All the values remained within the normal range for CSF with glare (Fig. 3).

The mean (SD) stereoacuity was 65 (57) (range, 40-400) s of arc at baseline, 51 (67) (range, 30-100) s of arc with the MF lens, and 105 (95) (range, 40-400) s of arc with the MV lens. The 49-s arc loss in stereoacuity with the MV lens compared with the MF lens was significant $(P<0.001)$.

The results of the subjective evaluation of visual comfort and handling ability are shown in Table 2 . The subjective scores did not differ significantly between the two corrections $(P>0.05)$. There was also no significant difference in the rating of subjective comfort between the lenses $(P>0.05)$ despite marginally better comfort with the MF lens, especially at the end of the day. There was also no significant difference in the overall satisfaction rating with subjective visual performance and subjective task performance between the two types of correction $(P>0.05)$.

\section{DISCUSSION}

This study confirmed that presbyopic patients can achieve 20/ 20 high-contrast distance and near VA or better with both lens types. The average high-contrast binocular VA for both lens types was, at most, two letters worse than the best-corrected spectacle VA and not significantly different. These findings were consistent with previous studies of MV and MF contact lenses, which reported a loss of less than one line of high-contrast VA. ${ }^{5,6}$

Our results for HCDVA were better than those of previous studies with the Proclear MF lens (Coopervision, Pleasanton, CA) (similar to the Biofinity MF design), whereas the HCNVA was comparable with previous results reported by Ferrer-Blasco and Madrid-Costa. ${ }^{8}$ Recently, Gupta et $a l,{ }^{5}$ in a study comparing MF 
232 Adaptation to Multifocal and Monovision Contact Lens Correction-Fernandes et al.

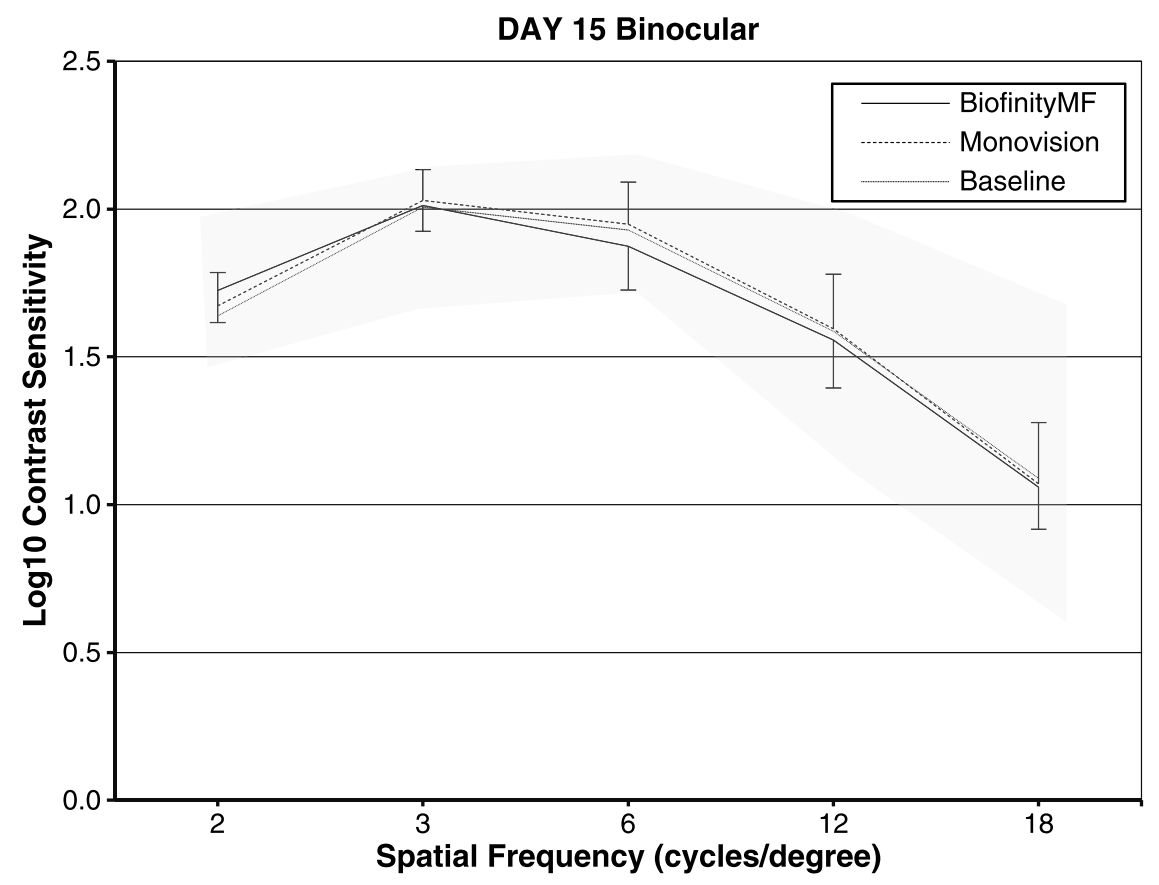

FIGURE 2.

The distance CSF values with the Biofinity MF contact lens and MV correction do not differ significantly ( $P>0.05$ at all spatial frequencies).

and MV correction, found that distance and near VA with a center-near aspheric simultaneous-vision MF contact lens (PureVision, Bauch \& Lomb, Rochester, NY) was worse than MV correction with a single-vision contact lens of the same material. The current sample was similar to that of Gupta et $\mathrm{al}^{5}$ regarding sample size, age, and measuring procedures; however, our results were different from those of Gupta et $\mathrm{al}^{5}$ who used a center-near aspheric MF lens in the dominant and nondominant eyes. In this study, we used an MF contact lens that combines different spherical and aspheric optics for the dominant and nondominant eyes. This makes a direct comparison difficult. The different designs might explain why, in this study, the MF vision performed better than MV, whereas Gupta et $\mathrm{al}^{5}$ reported the opposite. In their study, both eyes of the patient had the center part of the lens covered by the near power, which creates image degradation of far targets. In the case of the lens used in this study, this situation is only

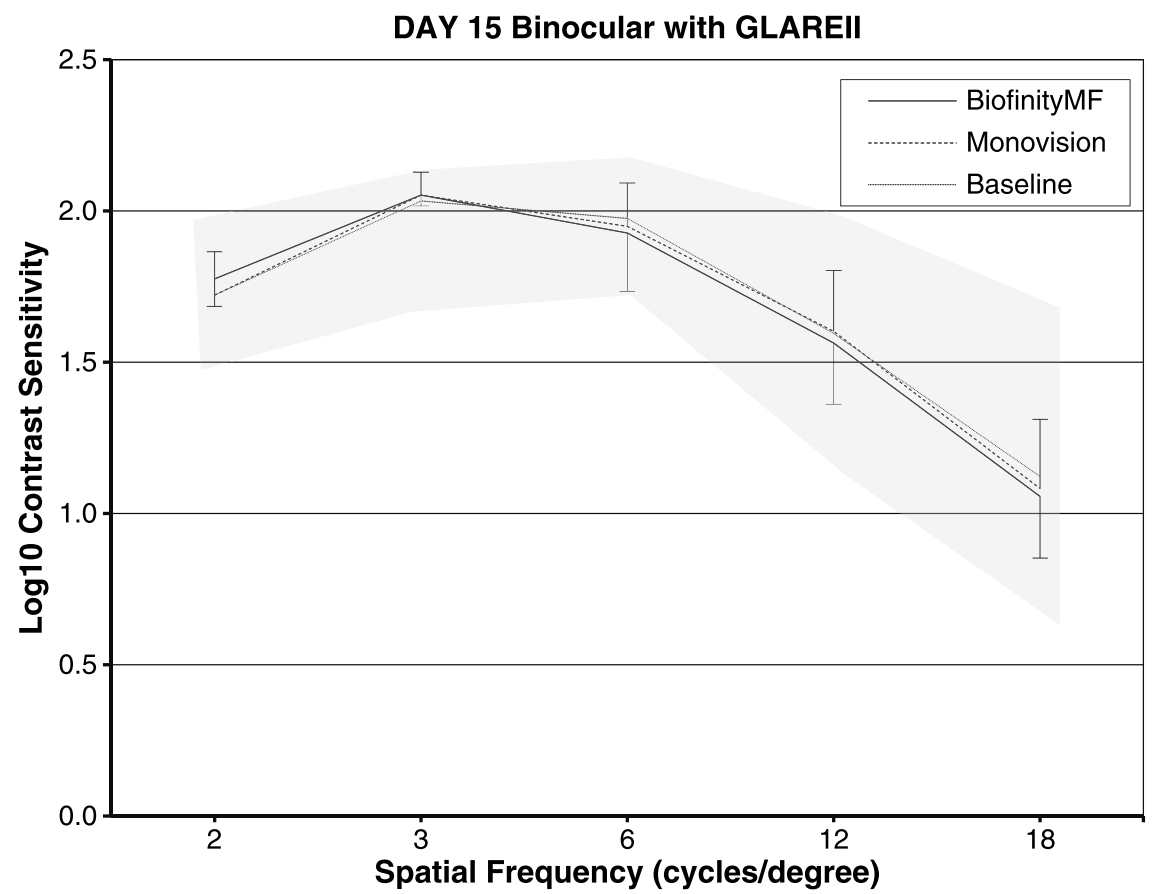

FIGURE 3.

The distance CSF with Glare II for the Biofinity MF contact lens and the MV correction do not differ significantly $(P>0.05$ for all spatial frequencies). 
TABLE 2.

Subjective evaluation of vision comfort and ability in multiple vertical visual analog scales questionnaire that enquires approximately three major aspects: subjective comfort and handling, subjective visual performance, and subjective task performance

\begin{tabular}{|c|c|c|c|c|}
\hline & & \multicolumn{3}{|c|}{ Mean (SD) } \\
\hline & & MF & MV & $P^{*}$ \\
\hline \multirow[t]{6}{*}{ Subjective handling and comfort } & Handling & $7.7(2.2)$ & $7.9(1.7)$ & 0.442 \\
\hline & Insertion & $6.4(2.7)$ & $7.5(1.8)$ & 0.033 \\
\hline & Removal & $6.9(2.7)$ & $7.5(1.7)$ & 0.491 \\
\hline & Comfort at insertion & $6.7(2.3)$ & $6.9(1.9)$ & 0.756 \\
\hline & Comfort during day & $6.9(1.9)$ & $6.7(1.8)$ & 0.643 \\
\hline & Comfort at the end of the day & $6.1(2.3)$ & $5.3(2.1)$ & 0.139 \\
\hline \multirow[t]{5}{*}{ Subjective visual performance (difficulty) } & Distance & $5.4(2.3)$ & $6.0(2.5)$ & 0.297 \\
\hline & Intermediate & $6.7(2.5)$ & $6.1(2.4)$ & 0.257 \\
\hline & Near & $6.7(2.1)$ & $6.2(2.2)$ & 0.667 \\
\hline & High luminance & $7.1(1.6)$ & $6.5(2.0)$ & 0.304 \\
\hline & Low luminance & $6.6(2.0)$ & $5.9(2.3)$ & 0.355 \\
\hline \multirow[t]{5}{*}{ Subjective visual performance (satisfaction) } & Distance & $5.8(2.2)$ & $6.3(2.7)$ & 0.214 \\
\hline & Intermediate & $6.7(1.7)$ & $6.6(2.1)$ & 0.979 \\
\hline & Near & $6.9(2.2)$ & $5.9(2.7)$ & 0.227 \\
\hline & High luminance & $6.9(1.8)$ & $6.6(2.1)$ & 0.959 \\
\hline & Low luminance & $6.3(1.9)$ & $6.2(2.5)$ & 0.952 \\
\hline \multirow[t]{10}{*}{ Subjective task performance } & Day driving & $7.6(2.6)$ & $8.0(1.6)$ & 0.736 \\
\hline & Night driving & $6.6(2.8)$ & $6.6(2.4)$ & 0.840 \\
\hline & Glare driving & $6.1(2.8)$ & $6.3(2.4)$ & 0.704 \\
\hline & Recognize faces & $6.6(1.9)$ & $6.6(2.4)$ & 0.940 \\
\hline & Computer work & $6.8(2.1)$ & $6.4(2.5)$ & 0.679 \\
\hline & Reading, intermediate & $6.7(1.9)$ & $6.9(1.6)$ & 0.522 \\
\hline & Reading, cellular & $7.5(2.2)$ & $7.1(2.1)$ & 0.330 \\
\hline & Reading, watch & $7.5(2.0)$ & $7.5(1.6)$ & 0.899 \\
\hline & Reading, correspondence & $7.4(2.0)$ & $7.4(1.7)$ & 0.753 \\
\hline & Fine manual works & $6.5(2.3)$ & $6.0(2.9)$ & 0.195 \\
\hline
\end{tabular}

The scale is 0 to 10 , with higher scores representing better condition.

At the end of the day, comfort was considerably better with MF than with MV system. Regarding the degree of satisfaction reported by subjective scores under different distances and viewing conditions, overall, MV performs better at distance, whereas intermediate and near vision is subjectively better with Biofinity MF.

*Overall significance with Wilcoxon signed-rank test.

present in the nondominant eye, whereas the dominant eye has a central spherical zone for clear distance vision. This might explain the better performance of the asymmetric MF lens designs in contrast to MV when compared with center-near MF lenses.

Furthermore, the asymmetric nature of the current design somewhat limits the distance vision in the nondominant eye and the near vision in the dominant eye, which is directly related to the central spherical $1.7-\mathrm{mm}$ near or $2.3-\mathrm{mm}$ distance areas, respectively. However, this study confirmed that a clinically and statistically significant improvement can be expected for approximately 15 days. It is important that clinicians consider this to prevent making substantial monocular changes in distance and near-lens power at an early stage of the fitting process.

Richdale et $\mathrm{al}^{6}$ reported results similar to this study for MV compared with MF soft contact lenses (SoftLens, Bausch \& Lomb, Rochester, NY). Those authors also measured high- and low-contrast distance and near logMAR VA using ETDRS charts, and the current results were within \pm 1 line of their reported VA for all experimental conditions except MV under near low-contrast VA, which performed better than the MF lens in their study.

Considering that MV induces significantly greater differences between the dominant and nondominant eyes compared with MF lenses, it is noteworthy to evaluate long-term behavior of the dominant and nondominant eyes and whether there is an adaptation process. Papas et $\mathrm{al}^{13}$ studied the short-term performance (4 days) of four different MF contact lens and reported significant changes only in clear vision at near and high-contrast near VA in low illumination. Those authors also stated that early assessment is relatively unrepresentative of performance later on during MF contact lens wear.

Regarding this, an interesting current observation was an improvement in distance and near VA from 1 to 15 days with the MF lens, whereas the patients maintained their VA or, in some cases, had worse VA with the MV correction. This was particularly significant for the nondominant eyes in which the MF distance VA significantly $(P=0.035)$ improved by one line after 15 days 
under high- and low-contrast conditions and also for near VA with a significant improvement in high- and low-contrast near VA in the nondominant eye and in low-contrast near VA in the dominant eye. This may explain the nonsignificant difference in the distance CSF between the two lens modalities after 15 days of wear, suggesting equal effects of increased retinal blur from superimposed images in simultaneous-vision (MF) contact lenses compared with the presence of a central blur suppression scotoma in MV. Gupta et $\mathrm{al}^{5}$ reported similar results for distance CSF using the VCTS 6500 by comparing MF contact lens and MV.

As expected, the stereoacuity was significantly poorer with MV than with the MF contact lens, which agreed with previously reported findings. ${ }^{5,6,8,14}$ This can be explained by the effects of monocular blurring produced by MV on stereoacuity, which is larger than the binocular defocus effects produced by retinal images superimposed on MF lenses. ${ }^{15}$ Furthermore, the values for this parameter in the MV group were scattered, and the differences prevailed even after 15 days of adaptation. Stereoacuity values for MF lenses after 15 days were similar to those obtained by previous authors with other MF lenses and with the Proclear MF lens with the same test. ${ }^{9}$

Subjective visual satisfaction and wearing success have been studied previously using different contact lens designs and wearing modalities. ${ }^{5,6,11,13,16}$ Papas et $\mathrm{al}^{13}$ found significant reductions in subjective visual satisfaction because of ghosting, halos, lens comfort, visual quality, visual fluctuation, facial recognition, and overall satisfaction with four MF soft contact lenses. Interestingly, those reductions were not associated with similar VA reductions, leading the authors to encourage subjective visual evaluation as a better indicator of lens performance than traditional VA tests. Similar results were observed in this study for the MF lens. However, despite the observed differences in visual function between the two presbyopic corrections in this study, there was no significant difference in the subjective perception of visual performance between the two lens types. The small sample may explain why some differences did not reach statistical significance. A larger study powered appropriately is needed to assess this.

The relatively lower scores regarding handling, insertion, and removal might be related to the lack of previous experience with contact lens wear, and the low comfort scores might be related as well. The end-of-day comfort was meaningfully better for the Biofinity MF than for the MV lens. Considering that both lenses are made of the same material and that similar levels of dehydration might be expected, the slightly better scores for end-of-day comfort observed with the MF lens might be related to the better visual adaptation and more effective MF correction with the MF contact lens.

The subjective visual scores showed a trend toward better distance vision with $\mathrm{MV}$ and better near vision with the MF lens. However, there were no significant differences; the logMAR VA outcomes did not justify these subjective outcomes. The reason for this lack of agreement might be the short adaptation period and the excellent distance vision that all patients had with their habitual correction before the contact lens trial.

Regarding several daily tasks such as driving, recognizing faces, visual tasks using different media among others, the comparative performance of the MV and MF lenses for intermediate visual tasks (computer work and reading at an intermediate distance) was somewhat surprising. This might be explained partially by the younger age of the patients and their residual amplitude of accommodation for viewing objects at an intermediate distance. This might be radically different for older patients with more advanced presbyopia. Recently, Gispets et $\mathrm{al}^{16}$ evaluated taskoriented visual satisfaction and wearing success with two types of simultaneous-vision MF soft contact lenses (Acuvue bifocal [Johnson \& Johnson, Jacksonville, FL] and Proclear MF lenses) and observed that visual satisfaction decreased for tasks involving higher visual demands for near and distance vision rather than for intermediate vision.

Regarding night-time driving, our results do not support the results of Chu et $\mathrm{al}^{17-19}$ who studied the effect of various presbyopic vision corrections on night-time driving. Those authors found that patients with MF contact lens showed a longer duration of recognition of distance targets and a smaller distance for recognition of traffic signs compared with patients with MV contact lens and that both groups are performing worse than those with single-vision or progressive-add spectacles. ${ }^{18}$ Although patients in this study reported poorer performance during night driving compared with during daytime driving, they did not show a subjective preference for MV or MF regarding their driving experience, even after having randomly experienced both methods of presbyopic correction for 15 days.

In conclusion, MF contact lenses provide satisfactory VA comparable with MV without compromising stereoacuity. There was no difference in comfort profiles between the MF and MV contact lenses made of the same material. When the objective and subjective results were compared, only the stereoacuity results indicated that MF contact lenses were significantly superior to MV lenses. As such, the simultaneous MF contact lens can potentially provide a better balance of real-world visual function because of minimal binocular disruption compared with $\mathrm{MV}$ lenses. An improvement in vision was observed from 1 to 15 days, which suggested an adaptation effect associated with the MF lens; the reverse was observed for the MV option.

\section{ACKNOWLEDGMENTS}

The authors declare that they do not have any proprietary or financial interest in any of the materials mentioned in this article. This study has been funded by projects PTDC/SAU-BEB/098392/2008 and PTDC/SAU-BEB/098391/ 2008 funded by the Portuguese Fundação para a Ciência e Tecnologia through the European Social Fund. This study has been partly supported by an unrestricted grant from CooperVision.

Received July 19, 2012; accepted November 28, 2012.

\section{REFERENCES}

1. Morgan PB, Efron N. Contact lens correction of presbyopia. Cont Lens Anterior Eye 2009;32:191-2.

2. Morgan PB, Efron N, Woods CA. An international survey of contact lens prescribing for presbyopia. Clin Exp Optom 2011;94:87-92.

3. Evans BJ. Monovision: a review. Ophthalmic Physiol Opt 2007;27: 417-39.

4. Bennett ES. Contact lens correction of presbyopia. Clin Exp Optom 2008;91:265-78.

5. Gupta N, Naroo SA, Wolffsohn JS. Visual comparison of multifocal contact lens to monovision. Optom Vis Sci 2009;86:98-105. 
6. Richdale K, Mitchell GL, Zadnik K. Comparison of multifocal and monovision soft contact lens corrections in patients with lowastigmatic presbyopia. Optom Vis Sci 2006;83:266-73.

7. McDonnell PJ, Lee P, Spritzer K, Lindblad AS, Hays RD. Associations of presbyopia with vision-targeted health-related quality of life. Arch Ophthalmol 2003;121:1577-81.

8. Ferrer-Blasco T, Madrid-Costa D. Stereoacuity with balanced presbyopic contact lenses. Clin Exp Optom 2011;94:76-81.

9. Ferrer-Blasco T, Madrid-Costa D. Stereoacuity with simultaneous vision multifocal contact lenses. Optom Vis Sci 2010;87:663-8.

10. Llorente-Guillemot A, Garcia-Lazaro S, Ferrer-Blasco T, PerezCambrodi RJ, Cervino A. Visual performance with simultaneous vision multifocal contact lenses. Clin Exp Optom 2012;95:54-9.

11. Situ P, Du Toit R, Fonn D, Simpson T. Successful monovision contact lens wearers refitted with bifocal contact lenses. Eye Contact Lens 2003;29:181-4.

12. Pesudovs K, Hazel CA, Doran RM, Elliott DB. The usefulness of Vistech and FACT contrast sensitivity charts for cataract and refractive surgery outcomes research. Br J Ophthalmol 2004;88:11-6.

13. Papas EB, Decenzo-Verbeten T, Fonn D, Holden BA, Kollbaum PS, Situ P, Tan J, Woods C. Utility of short-term evaluation of presbyopic contact lens performance. Eye Contact Lens 2009;35:144-8.

14. Rajagopalan AS, Bennett ES, Lakshminarayanan V. Visual performance of subjects wearing presbyopic contact lenses. Optom Vis Sci 2006;83:611-5.
15. Goodwin RT, Romano PE. Stereoacuity degradation by experimental and real monocular and binocular amblyopia. Invest Ophthalmol Vis Sci 1985;26:917-23.

16. Gispets J, Arjona M, Pujol J, Vilaseca M, Cardona G. Task oriented visual satisfaction and wearing success with two different simultaneous vision multifocal soft contact lenses. J Optom 2011;4:76-84.

17. Chu BS, Wood JM, Collins MJ. Influence of presbyopic corrections on driving-related eye and head movements. Optom Vis Sci 2009;86:1267-75.

18. Chu BS, Wood JM, Collins MJ. Effect of presbyopic vision corrections on perceptions of driving difficulty. Eye Contact Lens 2009;35:133-43.

19. Chu BS, Wood JM, Collins MJ. The effect of presbyopic vision corrections on nighttime driving performance. Invest Ophthalmol Vis Sci 2010;51:4861-6.

Paulo R.B. Fernandes Department of Physics (Optometry) School of Science, University of Minho 4710-057 Gualtar Braga, Portugal e-mail: pfernandes@fisica.uminho.pt 